resident population of about 250,000 . We provide child and adolescent services (for ages $0-18$ ) to the western half of Victoria which has a resident population of approximately two million.

Referrals have been generally by telephone although the trend is changing with more referrals being by letter. Traditionally the service has encouraged parents to contact the centre by phone. Cases are allocated at a weekly allocations meeting. The clinician then contacts the family by phone to arrange an appointment.

The survey includes patients referred who were offered an initial appointment. Of the 420 referrals, $68(16.2 \%)$ were either redirected to other agencies (30), did not require an appointment (32) or were not contactable on the phone (6) to arrange an appointment. We offered the remaining 352 an appointment and of this group 335 attended while 17 failed to keep the appointment. This gives a nonattendance rate of $4.8 \%$ and an attendance rate of $95.2 \%$. Eight out of the 17 patients who failed to attend were parent referrals.

Our attendance rate $(95.2 \%)$ is higher than the rate reported in comparable studies $(64 \%$ and 87.1\%) (Jaffa \& Griffins, 1990; Mathai \& Markantonakis, 1990).

The good attendance rate of first appointments in Melbourne appears to be due to the large number of parent referrals, i.e. $50 \%$ being made by parents directly to the service as opposed to $90 \%$ by general practitioners in the UK. It is recommended that parents be encouraged to contact the service directly along with the written referral being made by the GP or primary agency to the specialist service. Early parental contact not only facilitates information sharing about the clinic and its work but also ensures that the first appointment is kept by dealing with any apprehensions of the parent who has to attend the first appointment with the referred child. This is a cost-effective measure leading to saving of resources that could be easily wasted through failure to keep appointments.

JAFFA. T. \& GRIFFIN, S. (1990) Does a shorter wait for a first appointment improve the attendance rate in child psychiatry? Newsletter of the Association for Child Psychology \& Psychiatry, 12, 9-11.
MATHAJ, J. \& MARKANTONAKIS, A. (1990) Improving initial attendance to a child psychiatric clinic. Psychiatric Bulletin, 14, 151-152.

JoHN MATHAI, Royal Children's Hospital, Parkville, Victoria 3052, Australia

\section{Kufs disease: request for patients and families}

Sir: The neuronal ceroid lipofuscinoses (NCLs) are a group of inherited disorders characterised by the abnormal storage of lipopigments. They can be classified according to the clinical features and age of onset into four main subtypes: infantile, late infantile, juvenile and adult NCL. Adult onset NCL is also known as Kufs disease, and presents around the age of 30 years with dementia, myoclonic epilepsy and motor deterioration. Both autosomal recessive and dominant inheritance patterns have been described. Despite intensive biochemical investigation the underlying metabolic defect is unknown and treatment is symptomatic only.

The genes for infantile, one form of variant late infantile and juvenile NCL have now been mapped using family studies and linkage analysis. It is anticipated that these genes will be cloned within the near future and disease causing mutations characterised. The Department of Paediatrics at University College London has a long-standing interest in this group of disorders.

We plan to investigate families and individuals with Kufs disease using linkage analysis and mutation detection on a candidate gene basis. Ultimately the characterisation of this group of disorders at the molecular level will enable an understanding of the metabolic pathways involved, improved disease classification and genetic counselling, and may provide new therapeutic strategies.

Please contact for further information:

Ruth Williams (telephone: 0171-209-618, email rwilliam@hgmp.mrc.ac.uk) or SARA MOLE, (telephone: 0171-209-6104, email s.mole@ucl.ac.uk) Department of Paediatrics, UCLMS, The Rayne Institute, 5 University Street, London WC1E 6JJ (fax 0171-209-6103) 